(C2017, Elsevier. Licensed under the Creative Commons Attribution-NonCommercialNoDerivatives 4.0 International http://creativecommons.org/about/downloads

(9) $\odot \Theta \Theta$ 


\title{
Supporting people with young onset dementia and their families: An evaluation of a training course for care workers
}

\begin{abstract}
This article reports the findings of an evaluation of a training course for care workers who care for people with dementia in the community. Twenty-four care workers participated in the training which took place in London and Surrey, United Kingdom. The training had a significant positive impact on participants' confidence in understanding the experiences and social care needs of people with young onset dementia (YOD) and their families. Participants also perceived that the training would help them improve their working practice by furthering their understanding of practical approaches to supporting and caring for people with dementia in general. Additionally, participants reported many ways in which they perceived being able to specifically support and empower people with YOD. It was concluded that the short training course improved knowledge and confidence for care workers on dementia care, and specifically in understanding how to support people with YOD and their families. Dementia specific training should be considered by service managers as a way of potentially increasing care worker job satisfaction.
\end{abstract}




\section{Highlights}

- Care workers suggested the training would improve their working practice

- Care workers perceived gaining increased confidence in multiple aspects of dementia care

- Greatest increases in knowledge were seen in understanding issues affecting people with young onset dementia 


\section{Background}

Young onset dementia (YOD), also known as early onset dementia (Johannessen \& Moller, 2011; Chemali et al. 2012), is diagnosed in people aged 65 years or younger (Rossor et al. 2011). The number of people with YOD is difficult to determine primarily because many people present late with their symptoms (Jeffries \& Agrawal, 2009). However, in the United Kingdom (UK) it was estimated that 42,325 people had YOD in 2013, accounting for just over $5 \%$ of the total number of people living with the condition (Prince et al. 2013).

It is well known that caring for someone with dementia can be challenging (Brodaty et al. 2014; Ory et al. 2000; Svendsboe et al. 2016) and can affect the physical and mental health of carers (Pinquart \& Sörensen, 2007; Schulz \& Sherwood, 2009). However, there are specific difficulties for those caring for people with YOD. The evidence from a range of research studies suggests that family carers of people with YOD experience high levels of burden, stress, depression and psychosocial issues such as relationship problems, family conflict, employment and financial difficulties (van Vliet, 2010), which have a negative effect on their quality of life (Rossness et al. 2011). The long duration of caregiving coupled with the lack of specialist supportive services may also be a contributory factor to psychological distress reported by carers and families (Arai et al. 2007).

UK Government policy has attempted to address these concerns by improving workforce training. For example, it was identified that dementia training for health and social care workers should include information specific to YOD: "Training should enable an understanding of the diversity in dementia, including younger people with dementia..." (Department of Health [DH], 2009, p. 66). It is also recommended that health and social care workers adhere to seven common core principles for supporting people with dementia (DH, 2011). These include: promoting independence and encouraging activity; communicating sensitively to support meaningful interaction; recognising signs of distress; and ensuring family carers are valued, respected and supported.

Staff training is commonly seen as a way of improving the standard of care for people with dementia and their carers (Ballard et al. 2001; Kuske et al. 2007; Surr et al. 2016; Testad et al. 2016). Care staff's confidence in dealing with the behavioural and psychological 
symptoms of dementia (BPSD) has previously been shown to be low, for example evidence suggests only $29 \%$ of care staff feel confident in dealing with agitation or aggressive behaviour (Hughes et al. 2008). It was also reported in the same study that higher staff confidence in caring for people with dementia was associated with receiving dementia specific training. There is also evidence indicating that dementia care staff who receive ongoing training are more likely to report greater job satisfaction (Coogle et al. 2006; Sung et al. 2005; Zimmerman et al. 2005) and commitment to their role (Gurnik \& Hollis-Sawyer, 2003).

The evidence described above suggests that the care provided to people with YOD could be enhanced by training care staff about YOD. Such training could potentially enhance understanding of the similarities and differences between YOD and late onset dementia and also highlight the challenges for all stakeholders, including the person with YOD, their carers and those providing formal support.

This evaluation investigated a training course for care workers providing services to people with dementia. The training was delivered by a community interest organisation in the first half of 2015. The organisation provides training to health care workers who support people with dementia and their families (e.g. care workers; support workers and care home managers). The training course was structured to cover set topics and was delivered in the same way for each of the four training groups. The overall aim of the training was to improve participants' understanding of YOD and to help them provide enhanced support for people with YOD and their carers.

\section{Evaluation aims}

The aims of this evaluation were to:

1) Investigate the perceptions of the training on participants' confidence in working with people with YOD and their families.

2) Understand participants' perceptions of their learning and which aspects of the training were most important to facilitate understanding. 


\section{Methods}

\section{Setting}

The course was provided at five sites in and around London. Five courses took place between January and April 2015, with four running over two full days (either back to back or two weeks apart) and one running on four half days, one week apart. Participants were care workers based in the Greater London area. Courses were held in care homes or community buildings (such as the conference hall of a church).

\section{The training}

Training consisted of PowerPoint presentations, group work and video clips. It was led by a primary trainer (responsible for overseeing and delivering the training course), a support trainer (whose aim was to support the people with YOD and carer co-trainers) and by several people with YOD and their carers (referred to as co-trainers). The family carers and people with YOD talked to the participants about their personal experiences associated with YOD. They also assisted the primary trainer in delivering aspects of the course, for example, in helping to facilitate group discussions. The training was framed around Tom Kitwood's theory of applying person centred care in supporting people with dementia (Kitwood, 1997).

Participants were recruited by the training provider organisation team through contacting local care agencies and asking if they would be willing to ask their staff to attend. Care workers were eligible to attend the training if they were currently providing care for older people in the community.

\section{The questionnaires}

No validated rating scales were identified by the research team which would have measured the outcomes we were investigating. Subsequently, we constructed our own questionnaires. A mixed methods approach (Creswell, 2013; Hesse-Biber \& Burke Johnson, 2013) was utilised in developing two semi-structured questionnaires to determine whether the training aims had been achieved. The questionnaires were piloted on the first cohort of participants. Responses suggested that the face validity of the questionnaires was adequate for use in this population 
Questionnaire 1 had ten questions relating to confidence in working with people with dementia and these were Likert scales. For example, participants could choose from the following responses 'very confident, fairly confident, neither confident nor unconfident, unconfident or very unconfident'. Other questions were open-ended and allowed participants to respond in their own words. For example question $2 \mathrm{~b}$ : "If you have worked with anyone with young onset dementia, please describe the main challenges you found working with this group in the space below". Questionnaire 2 also included questions relating to the aspects of the course the participants found most useful, what they would change about the course and whether they would recommend the course to others. The questionnaires were designed to be brief with minimum impact on course delivery and took approximately 10 minute to complete.

Both questionnaires were anonymous and participants were asked to only use there initials or a pseudonym when completing them.

\section{Data collection}

A researcher external to the training provider organisation attended the beginning and end of each training course. The purpose of the evaluation was explained and what was required of participants was described. The importance giving their honest opinions was emphasised and they were encouraged to ask questions. It was also stressed to participants that: they were under no obligation to take part; their responses would be anonymised; they could withdraw at any time without explaining why and all data would be stored securely on a password protected University computer. Also, all participants were given an information sheet detailing the purpose of the evaluation and what their participation would involve. The information sheet included the researchers' contact details in case they had any questions at a later date. It was explained that the evaluation findings would be used for the development of one or more publications, but that no participants would be identifiable in these.

Questionnaire 1 was administered before the training began and included participants' demographic details, previous experience working with people with dementia, confidence in 
working with people with dementia, and perceived confidence specifically relating to working with people with YOD. Questionnaire 2 was administered at the end of the final training day and included many similar questions as questionnaire 1 in order to identify change. The course trainers also recorded group feedback at the end of two training sessions. The researchers were given this anonymised group feedback and this was also utilised in the qualitative data analysis. Consent was assumed on completion of the anonymous evaluation forms.

\section{Data analysis}

Quantitative data, including the participants' descriptive statistics, were analysed using SPSS version 22. Statistical significance was tested for using the Wilcoxon signed rank test. This non-parametric test was chosen as repeated measurements were conducted on the same sample. Qualitative data were content analysed, which involved developing themes based on the number of codes occurring in the data (Krippendorff, 1989). This was initially completed by one researcher (RS), with a second researcher checking whether the codes fitted the data (NG).

\section{Ethics}

The Faculty of Health, Social Care and Education, Kingston University research ethics committee were consulted prior to the evaluation. The committee follows the Health Research Authority (HRA) guidelines (Health Research Authority, 2016) regarding whether a project is considered research or evaluation and therefore whether or not it requires ethical approval. Following the HRA guidance, the chairs of the faculty ethics committee concluded that ethics approval was not required for this evaluation.

\section{Results}

\section{Participant characteristics}

In total, 22 out of 24 participants completed the full training course. The majority were female (96\%) and aged between 40 and 49 years (33\%). Over half (54\%) of the 24 participants who started the training had English as their first language, with almost a third (29\%) having other European languages and 13\% Asian languages as their first language. More than half spoke a second language, with the largest number of these speaking a 
European language other than English (29\%). The majority of the participants described themselves as either paid carers (58\%) or support workers (25\%). All were currently working with people with dementia and many had previously cared for a person with YOD. Full participant demographic details are available in Table 1.

\section{Quantitative results}

\section{Impact of the training on participant knowledge and understanding}

Prior to training, participants reported lacking knowledge and confidence in most areas related to the care of people with dementia. Participants reported increases in all areas after training (Table 2). The largest increases in knowledge were related to understanding the experiences and social care needs of people with YOD and their carers (questions 6, 7, 8 and 9). There was also a large increase in perceived confidence regarding describing the different approaches and activities used when working with people with YOD (question 10). Answers to all questions showed statistically significant increases in confidence $(p=<0.05)$.

The smallest increase in confidence in understanding the impact of dementia on families was observed with understanding the experiences of a person with dementia and their families in general, with the majority of participants indicating they had sufficient knowledge before the training.

$\underline{\text { Table } 1}$ about here

$\underline{\text { Table } 2}$ about here

\section{Qualitative findings}

Qualitative data were categorised into five themes:

1. Improvements in dementia knowledge;

2. Improved confidence in supporting and working with people with dementia in general; 
3. Improved confidence in supporting and working specifically with people with YOD;

4. Greater understanding of how to care for the families of people with YOD;

5. Importance of having carers and people with YOD as co-trainers on the course

Anonymous participant quotes (from questionnaires and group feedback) are presented to illustrate examples relating to these themes. All participants who responded to the quantitative questions also expanded with qualitative explanations.

\section{Improvements in dementia knowledge}

The participants described wide ranging increases in understanding, awareness and knowledge about dementia generally as a result of taking part in the training. These were similar to the findings on the quantitative scales. Participants' knowledge in understanding how people with YOD and their families are affected by dementia increased most, for example:

"I have learned how young onset dementia can affect the person and their families and that it can change their lives and roles." - Sarah (care worker)

An increase in knowledge specifically surrounding YOD was also apparent, with greater understanding of the associated signs and symptoms when compared to dementia generally. For example:

"I learned the differences between young onset dementia and dementia in older people and the impact on their lives. I learned how different parts of the brain affect the person's abilities." - Melanie (care worker)

Improved insight in how to support people with dementia in general was reported. Some participants also perceived specifically gaining an understanding of how to support people with YOD, as a participant commented:

"The course has given me more knowledge and awareness which will enable me to work in a more effective way with (people with) young onset dementia." - Katie (support worker) 


\section{Improved confidence in supporting and working with people with dementia in general}

Participants suggested the training would have a positive impact on how they would work with people with dementia in the future. In particular, just over half (13) commented on how it was expected to improve the way in which they support and care for people with dementia, including: improved listening, having more patience, treating people with dementia as individuals, promoting independence, including people with dementia in decision making and planning ahead. As one participant mentioned:

"It provided me with (the) understanding and information required to fully plan and support the care for a person living with dementia." - Pamela (project development worker)

Other ways in which the participants suggested training would improve how they work with people with dementia included: having greater awareness, knowledge, understanding and information about dementia (mentioned by seven participants) and improving the way in which they support carers (three participants), which was facilitated by being more aware of the needs and the challenges they encounter (three). Further, one participant, who attended the training over four days, explained how she was putting into practice her learning between training days:

"I have already been putting all I have learned into practice with my customer with dementia and (I am) getting a positive result." - Melanie (care worker)

Participants also reported improved knowledge of the Mental Capacity Act (DH, 2005) and its uses. For example, prior to training 13 participants reported they had no knowledge of the MCA or its use. This dropped to one after training. Similarly, only five participants understood one of the uses of the MCA was to protect or safeguard the rights of vulnerable people or those who lack capacity before training. This increased to 14 after training. 


\section{Improved confidence in supporting and working specifically with people with YOD}

In addition to the ways in which the training was perceived to have a positive impact on how participants may work with people with dementia generally, it also had a positive impact specifically on how they would work with people with YOD. Participants suggested it would improve the way in which they support, care for and empower people with YOD, including: giving adequate time; being more patient; improve their communication; being more empathetic; involving people with YOD in decision making; promoting independence; and helping people with YOD feel respected and valued. This was described by one participant, who commented:

"I will think how I can help them live in the moment while maintaining their sense of achievement and purpose. Maintaining vitality through my support as much as possible whilst bolstering independence."- Marcus (care worker)

Other ways in which participants suggested training may change how they work with people with YOD included: being better able to support families due to increased knowledge and awareness of its impact and a better understanding of the requirements and needs of people with YOD. This also included being more aware of providing age appropriate activities and involving people with YOD in service development, for example:

"I can understand the needs of a person with YOD more precisely. My vision is clearer in terms of respecting their needs and dignity." - Ruth (support worker)

"It has changed my understanding, approach and awareness of the young onset dementia. I am more confident and feel more capable and knowledgeable to work in a more effective way for the person with young onset dementia and their family. That is what this course has given me" - Taken from audio recorded group feedback after training.

\section{Improved understanding of how to care for the families of people with YOD}

Prior to training, five participants (13\%) were unsure how to support families of people with dementia in general and suggested this was a main challenge. Participants reported that this new knowledge has made them more sensitive to the families' requirements and more aware of families support needs. For example: 
"I understand the family needs support as much as the person with YOD. I will have to show more patience and respect for their needs and requirements." - Ruth (support worker)

Participants also reported that in the future they would be more mindful of including families in decision making and provide them with more information about other support services. As this participant mentioned:

"I will now be able to offer information and possibly provide support in ways of looking for support groups and organisations they can access as well as being inclusive for the cared for." - Pamela (project development worker)

These changes in caring for families are coupled with the previously documented increases in confidence in understanding the experiences of families of people with YOD (Table 2).

\section{Importance of having carers and people with YOD as co-trainers on the course}

The overwhelming majority found the presence of the person with YOD and the family carers as most useful in helping them to understand the experiences and challenges they encounter. Sixteen participants commented that the presence of the carers was most useful, with twelve highlighting the presence of the person with YOD was most useful. One participant relayed back to the group after training how she found the presence of a carer very beneficial in aiding her ability to understand the impact of YOD on families:

"I found it very informative and very useful that (the carer) was here to share his story because usually we ... in most of the cases usually we come across families who have their relatives who have dementia in their older age, whereas in (the carer's) situation it was a young onset dementia and to hear his side of the story and how he had to cope with it, it was very informative. That information we took from (the carer) today, we could actually use it somewhere in the future." - Taken from audio recorded group feedback after training. 


\section{Discussion}

This evaluation investigated the impact of dementia training on care workers' confidence in working with people with YOD and their families. It also aimed to understand their perceptions of the process of the training. The findings showed the training had a significant positive impact on participants' confidence in understanding the experiences and social care needs of people with YOD and their families. Participants also suggested that the training would help them improve their working practice by furthering their understanding of the practical approaches to supporting and caring for people with dementia. Further, participants reported many ways in which they described being able to specifically support and empower people with YOD. The reported increases in knowledge were perceived to have been helped by people with YOD and family carers being part of the course delivery and sharing their personal stories.

These findings suggest the training achieved its aims of improving participants' understanding of YOD and to potentially help them offer enhanced support for people with YOD and their carers. It supports evidence by Hughes et al. (2008) who reported higher staff confidence in caring for people with dementia was associated with being in receipt of dementia specific training. Additionally, the training provided participants with a grounding in the seven common core principles for supporting people with dementia as described by the DH (2011). Most notably: promoting independence and encouraging activity; communicating sensitively to support meaningful interaction; recognising signs of distress; and ensure family carers are valued respected and supported. These findings are important, as previous research has shown people with dementia and their carers often feel service providers lack an understanding of dementia (Prorok et al. 2013).

The qualitative findings also showed participants had been putting into practice their learning and had been seeing positive results. This has implications for job satisfaction, as research findings from various formal caregiving contexts has shown dementia care staff who receive ongoing training are more likely to report greater job satisfaction (Coogle et al. 2006; Sung et al. 2005; Zimmerman et al. 2005). It also shows how the training was having an impact on the care for people with dementia, something which staff training has previously shown improves standards of care (Ballard et al. 2001). 


\section{Strengths and limitations}

A strength of this evaluation is the questionnaire design which used a mixed methods approach (Creswell, 2013; Hesse-Biber \& Burke Johnson, 2013) to gain a more comprehensive understanding of the impact of the training (Mertens \& Hesse-Biber, 2012). For example, it enabled the results from the rating scales to be understood in terms of how participants perceive the training might impact upon their work. However, this evaluation has limitations which affect the generalisability of the findings to other training courses and health care workers. It is unclear whether different findings would be observed if all the participants had the same job role. For example, all carers or all support workers. Secondly, this evaluation suffers from its small sample size which weakens the interpretation of the results and limits the strengths of the conclusions drawn. Third, the practicalities of the data collection meant that the time scale between participants completing questionnaires 1 and 2 was relatively short. Leaving more time between data collection time points may have given a truer reflection of participants' retention of their learning.

\section{Recommendations and future research}

The recommendations for services providers are twofold. Firstly, that they should take steps to make sure their staff are adequately trained in dementia care generally, as a surprising finding from this evaluation was that only around half of participants were confident in understanding the signs, symptoms and causes of dementia in general. Second, the training service provider's commission for their staff should include, at least partially, information relating specifically to people with YOD and their carers.

Future evaluations of similar training courses should include the perceptions of carers and people with dementia on the impact of the training on the quality of care received. This would help identify whether the training has improved the experiences of those receiving care services. Further, re-evaluating the participants' knowledge after a time period of working practice would help identify if the knowledge gained is being maintained. 


\section{Conclusions}

This evaluation has not only demonstrated the need for dementia training in general, but that training should include information surrounding YOD and its impact on families, as few health and social care workers had specific confidence in understanding this prior to training. Participants perceived gaining increased confidence in multiple aspects of dementia knowledge. In particular, the greatest increases in knowledge were seen in understanding of issues specifically affecting people with YOD and their carers. Adequate training for formal carers working with people with dementia generally, as well as those working with people with YOD, should be considered by service managers as a way of potentially increasing client satisfaction.

\section{References}

Arai, A., Matsumoto, T., Manabu Ikeda, M., \& Arai Y. (2007) Do family caregivers perceive more difficulty when they look after patients with early onset dementia compared to those with late onset dementia? International Journal of Geriatric Psychiatry, 22: 1255-1261.

Ballard, C.G., O’Brien, J., James, I., \& Swann, A. (2001) Training carers in behavioural management skills. In C.G. Ballard, J. O’Brien, I. James, \& A. Swann (Eds.), Managing behavioural and psychological symptoms in people with dementia (pp. 169-193). Oxford: Oxford University Press.

Brodaty, H., Woodward, M., Boundy, K., Ames, D., \& Balshaw, R. (2014). Prevalence and Predictors of Burden in Caregivers of People with Dementia. American Journal of Geriatric Psychiatry, 22(8), 756-765.

Chemali, Z., Schamber, S., Tarbi, E., Acar, D., \& Avila-Urizar, M. (2012) Diagnosing early onset dementia and then what? A frustrating system of aftercare resources. International Journal of General Medicine, 5: 81-86. 
Coogle, C.L., Head, C.A. \& Parham, I.A. (2006). The long-term care workforce crisis:

Dementia-care training influences on job satisfaction and career commitment. Educational Gerontology, 32(8), 611-631.

Creswell, J. W. (2013) Research Design: Qualitative, Quantitative, and Mixed Methods Approaches. California: Sage.

Department of Health. (2005) Mental Capacity Act. London: HMSO.

Department of Health. (2009) Living Well with Dementia: A National Dementia Strategy. London: Crown.

Department of Health. (2011) Common Core Principles for Supporting People with Dementia: A Guide to Training the Social Care and Health Workforce. London: Crown.

Gurnik, M., \& Hollis-Sawyer, L. (2003) Empowering assisted living frontline care staffs to better care for Alzheimer's and dementia residents, Ageing International, 28, 82-97.

Health Research Authority. (2016) Defining Research: Research Ethics Service guidance to help you decide if your project requires review by a Research Ethics Committee. London: HRA.

Hesse-Biber, S. \& Burke Johnson, R. (2013) Coming at thing differently: Future directions of possible engagement with mixed methods research. Journal of Mixed Methods Research, 7(2), 103-109.

Hughes, J., Bagley, H., Reilly, S., Burns, A., \& Challis, D. (2008) Care staff working with people with dementia: Training, knowledge and confidence. Dementia: The International Journal of Social Research and Practice, 7(2), 227-238.

Jeffries, K. \& Agrawal, J. (2009) Early-onset dementia. Advances in Psychiatric Treatment, 15: 380-388. 
Johannessen, A. \& Möller, A. (2011) Experiences of persons with early-onset dementia in everyday life: a qualitative study. Dementia: The International Journal of Social Research and Practice, 12(4): 410-424.

Kitwood, T.M. (1997) Dementia Reconsidered: the Person Comes First. Buckingham: Open University Press

Krippendorff, K. (1989) Content analysis. In E. Barnouw, G. Gerbner, W. Schramm, T. L. Worth, \& L. Gross (Eds.), International encyclopedia of communication (Vol. 1, pp. 403-407). New York, NY: Oxford University Press.

Kuske, B., Hann,s S., Luck, T., Angermeyer, M.C., Behrens, J \& Riedel-Heller, S G. (2007) Nursing home staff training in dementia care: a systematic review of evaluated programs. International Psychogeriatrics, 19(5), 818-841.

Mertens, D. M. \& Hesse-Biber, S. (2012).Triangulation and mixed methods research: provocative positions. Journal of Mixed Methods Research, 6, 75-79.

Ory, M.G., Yee, J.L., Tennstedt, S.L., \& Schulz, R. (2000) The Extent and Impact of Dementia Care, in Schulz, R. Handbook on dementia caregiving: Evidence-based interventions for family caregivers (eds). New York: Springer.

Pinquart, M. \& Sorensen, S. (2007) Correlates of physical health of informal caregivers: a meta-analysis. The Journals of Gerontology Series B: Psychological Sciences and Social Sciences, 62(2), 126-137.

Prince, M., Bryce, R., Albanese, E., Wimo, A., Ribeiro, W., Ferri, C.P. (2013) The global prevalence of dementia: A systematic review and meta-analysis. Alzheimer's \& Dementia, 9(1): 63-75.e2. 
Prorok, J.C., Horgan, S., \& Seitz, D.P. (2013) Health care experiences of people with dementia and their caregivers: a meta-ethnographic analysis of qualitative studies. Canadian Medical Association Journal, 185(14): E669-E680.

Rossor, M.N., Fox, N.C., Mummery, C.J., Schott, J.M., \& Warren, J.D. (2011) The diagnosis of young-onset dementia. Lancet Neurology, 9(8): 793-806.

Rosness, T.A., Mjorud, M., \& Engedal, K. (2011) Quality of life and depression in carers of patients with early onset dementia. Aging \& Mental Health, 15: 299-306.

Schulz, R. \& Sherwood, P.R. (2009) Physical and Mental Health Effects of Family Caregiving. American Journal of Nursing, 108(9), 23-27.

Sung, H.C., Chang, S.M. \& Tsai, C.S. (2005) Working in long-term care settings for older people with dementia: nurses' aides. - reports regular training helps nurses aids deal with difficulties in working with people with dementia and improves job satisfaction. Journal of Clinical Nursing, 14(5), 587-593.

Surr, C.A., Smith, S.J., Crossland, J., Robins, J. (2016) Impact of a person-centred dementia care training programme on hospital staff attitudes, role efficacy and perceptions of caring for people with dementia: A repeated measures study. International Journal of Nursing Studies, 53, 144-151.

Svendsboe, E., Terum, T., Testad, I., Aarsland, D., Ulstein, I., Corbet,t A. \& Rongve, A. (2016) Caregiver burden in family carers of people with dementia with Lewy bodies and Alzheimer's disease. International Journal of Geriatric Psychiatry, 31(9):1075-83.

Testad, I., Mekki, T.E., Førland, O., Øye C., Tveit, E.M., Jacobsen, F., \& Kirkevold, Ø. (2016) Modeling and evaluating evidence-based continuing education program in nursing home dementia care (MEDCED) - training of care home staff to reduce use of restraint in care home residents with dementia. A cluster randomized controlled trial. International Journal of Geriatric Psychiatry, 31(1), 24-132. 
van Vliet, D., de Vugt, M.E., Bakker, C., Koopmans, R.T., \& Verhey, F.R.J. (2010) Impact of early onset dementia on caregivers: A review. International Journal of Geriatric Psychiatry, 25: $1091-1100$.

Zimmerman, S., Williams, C.S., Reed, P.S., Boustani, M., Preisser, J.S., Heck, E., \& Sloane, P. D. (2005) Attitudes, stress, and satisfaction of staff who care for residents with dementia. Gerontologist, 1(1), 96-105. 


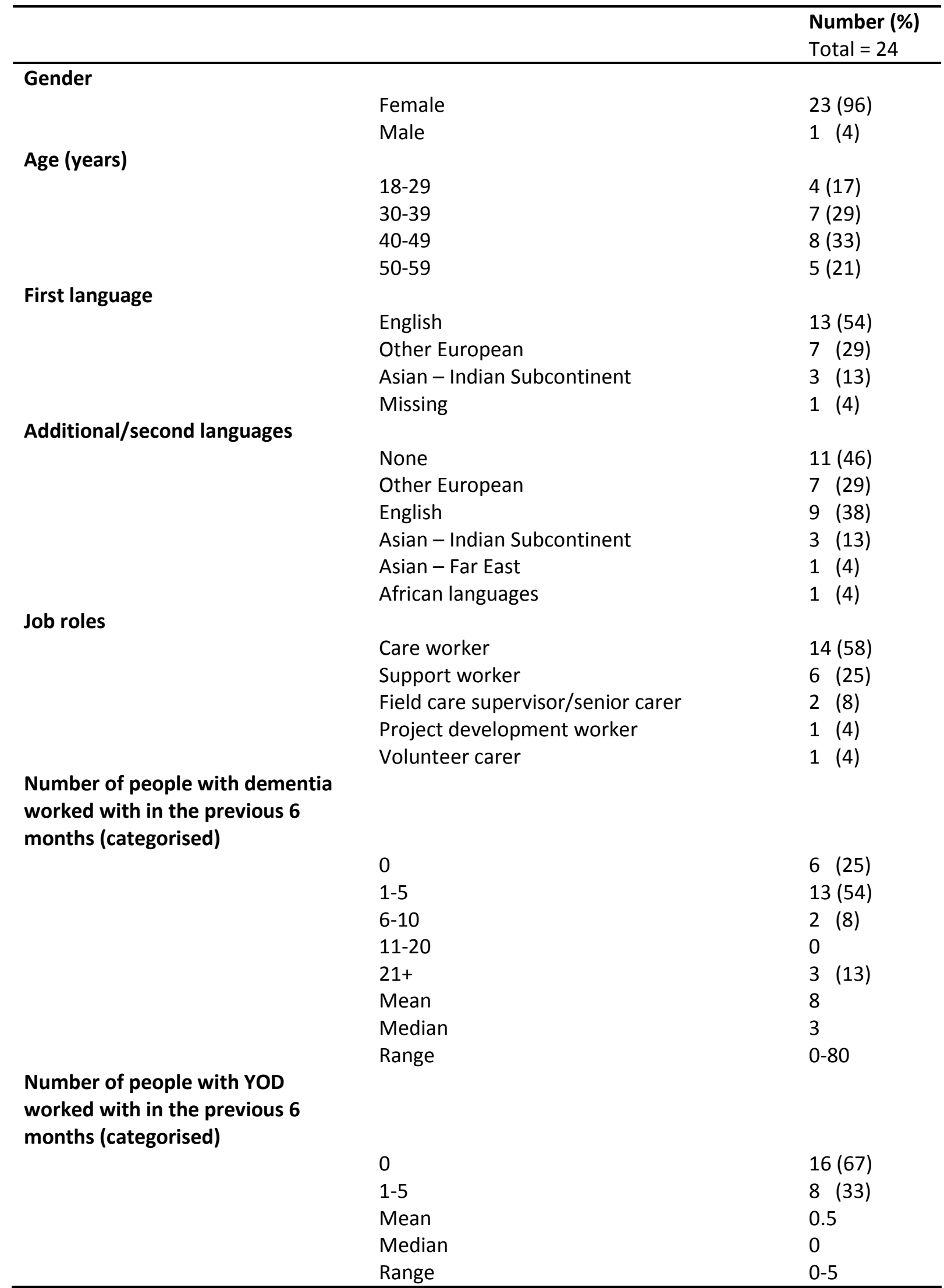


Table 2. Knowledge and confidence before and after training

\begin{tabular}{|c|c|c|c|c|}
\hline & Confidence in: & $\begin{array}{l}\text { Confident } \\
\text { before (\%) }\end{array}$ & $\begin{array}{l}\text { Confident } \\
\text { after* (\%) }\end{array}$ & $\begin{array}{l}\text { Significance } \\
\text { (Wilcoxon signed } \\
\text { rank test) }\end{array}$ \\
\hline 1 & Knowing causes of dementia (in general) & $11(46 \%)$ & $21(95 \%)$ & $p=0.002$ \\
\hline 2 & Knowing the signs and symptoms of dementia (in general) & $11(46 \%)$ & $22(100 \%)$ & $p=0.001$ \\
\hline 3 & Understanding the difficulties in diagnosing YOD & $6(25 \%)$ & $20(91 \%)$ & $p=<0.000$ \\
\hline 4 & Understanding the experiences of a person with dementia & $12(50 \%)$ & $21(95 \%)$ & $p=0.002$ \\
\hline 5 & Understanding the experiences of the family of a person with dementia & $11(46 \%)$ & $20(91 \%)$ & $p=0.002$ \\
\hline 6 & Understanding the experiences of a person with YOD & $2(8 \%)$ & $20(91 \%)$ & $p=<0.000$ \\
\hline 7 & Understanding the experiences of the family of a person with YOD & $2(8 \%)$ & $20(91 \%)$ & $p=<0.000$ \\
\hline 8 & Describing the different social care needs of a person with YOD & $1(4 \%)$ & $22(100 \%)$ & $p=<0.000$ \\
\hline 9 & Describing the different social care needs of the family of a person with YOD & 0 & $22(100 \%)$ & $p=<0.000$ \\
\hline 10 & $\begin{array}{l}\text { Describing different approaches and activities to be used when working with } \\
\text { a person with YOD }\end{array}$ & $1(4 \%)$ & $22(100 \%)$ & $p=<0.000$ \\
\hline
\end{tabular}

*Two participants missing from the follow-up data analysis 